\title{
ON POLYNOMIALS SATISFYING A TURÁN TYPE INEQUALITY
}

\author{
GEORGE CSORDAS AND JACK WILLIAMSON
}

\begin{abstract}
For Legendre polynomials $P_{n}(x)$, P. Turán has established the inequality

$$
\Delta_{n}(x)=P_{n}^{2}(x)-P_{n+1}(x) P_{n-1}(x) \geqq 0, \quad-1 \leqq x \leqq 1, n \geqq 1,
$$

with equality only for $x= \pm 1$. This inequality has generated considerable interest, and analogous inequalities have been extended to various classes of polynomials: ultraspherical, Laguerre, Hermite, and a class of Jacobi polynomials. Our purpose here is to determine necessary and sufficient conditions for a general class of polynomials to satisfy a Turán type inequality and to characterize the generating functions of such a class.
\end{abstract}

1. Introduction. In 1948, Szegö [12] called attention to the following remarkable inequality of $\mathrm{P}$. Turán for Legendre polynomials $P_{n}(x)$ :

$$
\Delta_{n}(x)=P_{n}^{2}(x)-P_{n+1}(x) P_{n-1}(x) \geqq 0, \quad-1 \leqq x \leqq 1, n \geqq 1,
$$

with equality only for $x= \pm 1$. This inequality has generated considerable interest (see, e.g., [4] and [10]). Turán's proof and three additional proofs of (1.1) were given by Szegö [12], who also extended the result to ultraspherical, Laguerre, and Hermite polynomials. More recently, Gasper [2] proved the analogue of (1.1) for a class of Jacobi polynomials. Our purpose here is to determine necessary and sufficient conditions for a general class of polynomials to satisfy a Turán type inequality and to characterize the generating functions of such a class.

Let $\left\{a_{k}\right\}_{k=0}^{\infty}$ be a sequence of real numbers with $a_{0}=1$, let

$$
g_{n}(x)=\sum_{k=0}^{n}\left(\begin{array}{l}
n \\
k
\end{array}\right) a_{k} x^{k}
$$

and let

$$
\Delta_{n}(x)=g_{n}^{2}(x)-g_{n+1}(x) g_{n-1}(x), \quad n \geqq 1 .
$$

Received by the editors June 4, 1973.

AMS (MOS) subject classifications (1970). Primary 26A75, 30A08, 33A70; Secondary 30A64, 30A74.

Key words and phrases. Inequality, Turán type inequality, real, simple zeros, generating functions, entire functions. 
If, for each $x,-\infty<x<\infty$, either

$$
\Delta_{n}(x)>0, \quad n \geqq 1, \quad \text { or } \quad \Delta_{n}(x)=0, \quad n \geqq 1,
$$

then we shall say that the sequence $\left\{g_{n}\right\}_{n=0}^{\infty}$ satisfies a Turán type inequality.

J. L. Burchnall [1] showed that if $g_{n}(x)$ has real, simple zeros for $n \geqq 1$, then $\left\{g_{n}\right\}$ satisfies condition (T). In addition, it is easy to see that if

$$
g_{n}(x)=\left(1+a_{1} x\right)^{n}, \quad n \geqq 1,
$$

then $\Delta_{n}(x) \equiv 0, n \geqq 1$, so that the sequence $\left\{g_{n}\right\}$ defined by (1.3) trivially satisfies condition $(T)$.

Now it is natural to inquire whether there are other examples of polynomials of the form (1.2) which satisfy a Turán type inequality. By way of an answer to this question, we shall show that provided the coefficients $\left\{a_{k}\right\}$ satisfy a mild restriction, the two sequences of polynomials mentioned above are the only sequences which satisfy a Turán type inequality, that is, satisfy condition (T). Indeed, if $\left\{g_{n}\right\}$ is a sequence of polynomials defined by (1.2), then we have

THEOREM 1. If $\left\{g_{n}\right\}$ satisfies condition (T) and if $\Delta_{n}(\xi)=0$ for some $\xi \neq 0, n \geqq 1$, then

$$
g_{n}(x)=\left(1+a_{1} x\right)^{n}, \quad n \geqq 1 .
$$

THEOREM 2. If $\Delta_{n}(x)>0$ for all $x \neq 0, n \geqq 1$, and if the sequence of coefficients $\left\{a_{k}\right\}$ satisfies the condition

$$
a_{k-1} a_{k+1}<0 \text { whenever } a_{k}=0,
$$

then $g_{n}(x)$ has real, simple zeros for $n \geqq 1$.

We remark that it is not difficult to construct examples which show that Theorem 2 is false if condition (1.4) is omitted.

2. Proof of Theorem 1. If $\Delta_{n}(\xi)=0$ for some $\xi \neq 0, n \geqq 1$, then, in particular, $\Delta_{1}(\xi)=\left(a_{1}^{2}-a_{0} a_{2}\right) \xi^{2}=0$. Hence, $a_{1}^{2}-a_{0} a_{2}=0$ and $a$ fortiori $\Delta_{1}(x)=\left(a_{1}^{2}-a_{0} a_{2}\right) x^{2} \equiv 0$. But then, in view of condition $(\mathrm{T})$,

$$
\Delta_{n}(x) \equiv 0, \quad n=1,2, \cdots .
$$

Now $\Delta_{n}(x)$ is a polynomial of degree $2 n$ with leading coefficient $a_{n}^{2}-a_{n-1} a_{n+1}$, so (2.1) implies that

$$
a_{n}^{2}-a_{n-1} a_{n+1}=0, \quad n=1,2, \cdots .
$$

Thus, it follows from (2.2) and an easy induction argument that $a_{n}=a_{1}^{n}$, $n=1,2, \cdots$. Hence,

$$
g_{n}(x)=\sum_{k=0}^{n}\left(\begin{array}{l}
n \\
k
\end{array}\right) a_{k} x^{k}=\sum_{k=0}^{n}\left(\begin{array}{l}
n \\
k
\end{array}\right) a_{1}^{k} x^{k}=\left(1+a_{1} x\right)^{n} .
$$


3. Proof of Theorem 2. The proof of Theorem 2 depends upon an algebraic rule, which Pólya [6, p. 21] credits to de Gua, and a lemma.

de Gua's rule. A polynomial $f(x)$ with real coefficients has real, simple zeros only, if its derivatives $f^{\prime}(x), f^{\prime \prime}(x), \cdots, f^{(n)}(x), \cdots$ have the property:

If $\xi$ is real and $f^{(n)}(\xi)=0$, then $f^{(n-1)}(\xi) f^{(n+1)}(\xi)<0$.

Lemma. Under the hypothesis of Theorem 2,

$$
a_{n}^{2}-a_{n-1} a_{n+1}>0, \quad n=1,2, \cdots .
$$

Proof. The proof will be by induction. First observe that, by hypothesis, $\Delta_{1}(x)=\left(a_{1}^{2}-a_{0} a_{2}\right) x^{2}>0, x \neq 0$, and hence,

$$
a_{1}^{2}-a_{0} a_{2}>0 \text {. }
$$

Now suppose

$$
a_{1}^{2}-a_{0} a_{2}>0, \quad a_{2}^{2}-a_{1} a_{3}>0, \cdots, \quad a_{n-1}^{2}-a_{n-2} a_{n}>0 .
$$

To show that $a_{n}^{2}-a_{n-1} a_{n+1}>0$, note that

$$
\Delta_{n}(x)=\sum_{k=2}^{2 n} c_{k} x^{k}
$$

where

$$
c_{2 n}=a_{n}^{2}-a_{n-1} a_{n+1} \quad \text { and } \quad c_{2 n-1}=(n-1)\left(a_{n} a_{n-1}-a_{n-2} a_{n+1}\right) .
$$

Thus, the hypothesis $\Delta_{n}(x)>0, x \neq 0$, implies $a_{n}^{2}-a_{n-1} a_{n+1} \geqq 0$ and

$$
a_{n} a_{n-1}=a_{n-2} a_{n+1} \quad \text { whenever } a_{n}^{2}-a_{n-1} a_{n+1}=0 \text {. }
$$

Now if $a_{n}=0$, then it follows from (1.4) that $a_{n}^{2}-a_{n-1} a_{n+1}>0$. If, on the other hand, $a_{n} \neq 0$ and $a_{n}^{2}-a_{n-1} a_{n+1}=0$, then (3.3) implies $a_{n} a_{n-1}=$ $a_{n-2} a_{n+1}$. Consequently, it follows that $a_{n-1}^{2}-a_{n-2} a_{n}=0$. This contradicts (3.2) and thus, the induction is complete.

We now proceed with the proof of Theorem 2. First, set

$$
P_{n}(x)=(1 / n !) x^{n} g_{n}\left(x^{-1}\right)
$$

and observe that

$$
P_{n}^{\prime}(x)=P_{n-1}(x) .
$$

Next, express $x^{2 n} \Delta_{n}\left(x^{-1}\right)$ in terms of the polynomials defined by (3.4) to obtain

$$
x^{2 n} \Delta_{n}\left(x^{-1}\right)=(n+1) !(n-1) !\left[\frac{n}{n+1} P_{n}^{2}(x)-P_{n-1}(x) P_{n+1}(x)\right]
$$


Since by hypothesis $\Delta_{n}(x)>0$ for $x \neq 0, n \geqq 1,(3.6)$ implies

$$
\sigma_{n}(x)=\frac{n}{n+1} P_{n}^{2}(x)-P_{n-1}(x) P_{n+1}(x)>0, \quad x \neq 0, n \geqq 1 .
$$

Moreover, the preceding lemma implies

$$
(n-1) !(n+1) ! \sigma_{n}(0)=a_{n}^{2}-a_{n-1} a_{n+1}>0, \quad n \geqq 1 .
$$

Thus, by (3.7) and (3.8), we have

$$
\sigma_{n}(x)>0, \quad-\infty<x<\infty, \quad n \geqq 1 .
$$

Now suppose that $P_{n}^{(k)}(\xi)=0$. Then by (3.5) and (3.9), we have

$$
\begin{aligned}
0<\sigma_{n-k}(\xi) & =\frac{n-k}{n-k+1} P_{n-k}^{2}(\xi)-P_{n-k+1}(\xi) P_{n-k-1}(\xi) \\
& =\frac{n-k}{n-k+1}\left[P_{n}^{(k)}(\xi)\right]^{2}-P_{n}^{(k-1)}(\xi) P_{n}^{(k+1)}(\xi) \\
& =-P_{n}^{(k-1)}(\xi) P_{n}^{(k+1)}(\xi) .
\end{aligned}
$$

Thus,

$$
P_{n}^{(k-1)}(\xi) P_{n}^{(k+1)}(\xi)<0, \quad k=1, \cdots, n-1,
$$

and de Gua's rule implies $P_{n}(x)$ has real, simple zeros for $n \geqq 1$. Since $g_{n}(x)=n ! x^{-n} P_{n}\left(x^{-1}\right)$, it follows that $g_{n}(x)$ has real, simple zeros for $n \geqq 1$.

Theorem 2 has the following immediate but interesting

CoROLlaRY. Let $\Delta_{n}(x)$ and $\left\{a_{n}\right\}$ satisfy the hypothesis of Theorem 2 and set

$$
g_{n, p}(x)=\sum_{k=0}^{n}\left(\begin{array}{l}
n \\
k
\end{array}\right) a_{k+p} x^{k}, \quad n \geqq 1, p \geqq 1 .
$$

Then $g_{n, p}(x)$ has real, simple zeros and, for every $p \geqq 1$, the sequence $\left\{g_{n, p}\right\}$ satisfies condition $(\mathrm{T})$.

Proof. Since

$$
g_{n, p}(x)=\frac{p !}{(n+p) !} g_{n+p}^{(p)}(x)
$$

and $g_{n+p}(x)$ has real, simple zeros, Rolle's theorem implies that $g_{n, p}(x)$ has real, simple zeros. The assertion that $\left\{g_{n, p}\right\}$ satisfies condition (T), for $p \geqq 1$, then follows from Burchnall's result mentioned in the Introduction. 
4. The generating functions of polynomials satisfying condition (T). Suppose

$$
f(z)=\sum_{k=0}^{\infty} a_{k} z^{k} / k ! \quad\left(a_{0}=1\right)
$$

is holomorphic in a neighborhood of the origin. It is well known (see, e.g., [8]) that the sequence of polynomials $\left\{g_{n}\right\}$, defined by (1.2), is generated by $e^{z} f(x z)$, that is, $e^{z} f(x z)=\sum_{n=0}^{\infty} g_{n}(x) z^{n} / n$ !, while the sequence of polynomials $\left\{P_{n}\right\}$, defined by (3.4), is generated by $e^{x z} f(z)$, that is, $e^{x z} f(z)=\sum_{n=0}^{\infty} P_{n}(x) z^{n}$. (The polynomials $P_{n}(x)$ are called Appell polynomials.)

Of special interest is the case when $f(z)$ is of the form

$$
f(z)=e^{-\gamma z^{2}+\beta z} \prod_{n=1}^{\infty}\left(1-z / z_{n}\right) e^{z / z_{n}}
$$

where $\gamma \geqq 0, \beta, z_{n}$ are real and $\sum_{n=1}^{\infty} z_{n}^{-2}<\infty$.

We shall say that an entire function $f(z)$ of the form (4.2) belongs to the class $\mathscr{L}-\mathscr{P}$ (Laguerre-Pólya) and we shall write $f(z) \in \mathscr{L}-\mathscr{P}$.

If $f(z) \in \mathscr{L}-\mathscr{P}$ is given by (4.1), then it is well known [7, p. 110] that, for $n \geqq 1, g_{n}(x)=\sum_{k=0}^{n}\left(\begin{array}{l}n \\ k\end{array}\right) a_{k} x^{k}$ has only real zeros. Consequently, it follows $([3, \S 4.3]$ or $[9$, p. 76$])$ that

$$
a_{k}^{2}-a_{k-1} a_{k+1}>0, \quad k \geqq 1, \quad \text { or } \quad a_{k}^{2}-a_{k-1} a_{k+1}=0, \quad k \geqq 1 .^{1}
$$

(Note that the second condition in (4.3) implies that $f(z)=e^{a_{1} z}$.) Since $f(z) \in \mathscr{L}-\mathscr{P}$ clearly implies $e^{z} f(x z) \in \mathscr{L}-\mathscr{P}$ and $e^{x z} f(z) \in \mathscr{L}-\mathscr{P}$ for every $x$, $-\infty<x<\infty$, the following proposition is a consequence of (4.3).

Proposition 1. Let $f(z)$ be given by (4.1). If $f(z) \in \mathscr{L}-\mathscr{P}$, then the polynomial sequences $\left\{g_{n}\right\}$ and $\left\{n ! P_{n}\right\}$ generated by $e^{z} f(x z)$ and $e^{x z} f(z)$ respectively, satisfy condition $(\mathrm{T})$.

Conversely, as a consequence of Theorem 2, we have

Proposition 2. If $\left\{a_{k}\right\}_{k=0}^{\infty}, a_{0}=1$, is a sequence of real numbers which satisfies (1.4) and if the sequence $\left\{g_{n}\right\}$ defined by (1.2) satisfies condition $(\mathrm{T})$ then the function

belongs to the class $\mathscr{L}-\mathscr{P}$.

$$
f(z)=\sum_{k=0}^{\infty} a_{k} z^{k} / k !
$$

${ }^{1}$ Szegö [12] used this condition to show that many of the classical polynomials satisfy a Turán type inequality. 
Proof. By Theorems 1 and $2, g_{n}(x), n \geqq 1$, has only real zeros, and hence, the polynomial $G_{n}(z), n \geqq 1$, where

$$
G_{n}(z)=g_{n}\left(\frac{z}{n}\right)=\sum_{k=0}^{n} \frac{a_{k}}{k !}\left(1-\frac{1}{n}\right) \cdots\left(1-\frac{k-1}{n}\right) z^{k}
$$

has only real zeros. Furthermore, since

$$
G_{n}(0)=1, \quad\left|G_{n}^{\prime}(0)\right|=\left|a_{1}\right| \text { and }\left|G_{n}^{\prime \prime}(0)\right| \leqq \frac{1}{2}\left|a_{2}\right|,
$$

it follows (see, e.g., Szász [11]) that $\left\{G_{n}(z)\right\}$ is a normal family. Now $f(z)$ is clearly the unique limit function of the sequence $\left\{G_{n}(z)\right\}$; thus, $\left\{G_{n}(z)\right\}$ converges uniformly to $f(z)$ on every compact subset of the plane. Since $G_{n}(z), n \geqq 1$, has only real zeros, a classical result of Pólya [5] implies that $f(z) \in \mathscr{L}-\mathscr{P}$.

\section{REFERENCES}

1. J. L. Burchnall, An algebraic property of the classical polynomials, Proc. London Math. Soc. (3) 1 (1951), 232-240. MR 13, 648.

2. G. Gasper, An inequality of Turán type for Jacobi polynomials, Proc. Amer. Math. Soc. 32 (1972), 435-439. MR 44 \#7013.

3. G. H. Hardy, J. E. Littlewood and G. Pólya, Inequalities, Cambridge Univ. Press, Cambridge, 1934.

4. S. Karlin and G. Szegö, On certain determinants whose elements are orthogonal polynomials, J. Analyse Math. 8 (1960/61), 1-157. MR 26 \#539.

5. G. Pólya, Uber Annäherung durch Polynome mit lauter reellen Wurzeln, Rend. Circ. Mat. Palermo 36 (1913), 279-295.

6. - Some problems connected with Fourier's work on transcendental equations, Quart. J. Math. Oxford Ser. 1 (1930), 21-34.

7. G. Pólya and J. Schur, Über zwei Arten von Faktorenfolgen in der Theorie der algebraischen Gleichungen, J. Reine Angew. Math. 144 (1914), 89-113.

8. E. D. Rainville, Certain generating functions and associated polynomials, Amer. Math. Monthly 52 (1945), 239-250. MR 6, 211.

9. J. Schur, Żwei Sätze über algebraische Gleichungen mit lauter reellen Wurzeln, J. Reine Angew. Math. 144 (1914), 75-88.

10. H. Skovgaard, On inequalities of the Turán type, Math. Scand. 2 (1954), 65-73. MR 16, 118.

11. O. Szász, On sequences of polynomials and the distribution of their zeros, Bull. Amer. Math. Soc. 49 (1943), 377-383. MR 4, 273.

12. G. Szegö, On an inequality of $P$. Turán concerning Legendre polynomials, Bull. Amer. Math. Soc. 54 (1948), 401-405. MR 9, 429.

Department of Mathematics, University of Hawail, Honolulu, Hawail 96822 